\title{
Reduced premature mortality in Rwanda: lessons from
} success

Some errors went uncorrected in the table of this article by Paul Farmer and colleagues (BMJ 2013;346:f65, doi:10.1136/bmj. f65). The figures for malaria were incorrect, the subheadings wrongly appeared at the top of the table, and the source references should have been $2,33,34,35$, and 36 . The correct table is here $\Downarrow$. In addition, the affiliation for Soline Dusabeyesu
Mugeni should be the Clinton Health Access Initiative [not Partners in Health].

Cite this as: BMJ 2013;346:f534

๑ BMJ Publishing Group Ltd 2013 


\section{Table}

Table | Premature mortality in Rwanda, 2000-11 23333536

Annual rate of reduction

20002011 Total reduction (\%) (\%) Regional rank Global rank ${ }^{*}$

\section{HIV/AIDS}

\begin{tabular}{|c|c|c|c|c|c|c|}
\hline HIV incidence/ 100000 & 207 & $88 \dagger$ & 57.5 & 9.5 & 5 & 8 \\
\hline AIDS deaths/100 000 & 190 & $41 \dagger$ & 78.4 & 17.0 & 1 & 1 \\
\hline \multicolumn{7}{|l|}{ Tuberculosis } \\
\hline Incidence/100 000 & 326 & 94 & 71.2 & 11.3 & 1 & 4 \\
\hline Mortality/100 000 & 48 & 11 & 77.1 & 13.4 & 1 & 5 \\
\hline \multicolumn{7}{|l|}{ Malaria } \\
\hline Total cases & $1654246 \ddagger$ & 208858 & 87.4 & 34.45 & NA & NA \\
\hline Total deaths & $2581 \ddagger$ & 380 & 85.3 & 31.9 & NA & NA \\
\hline \multicolumn{7}{|l|}{ Child and maternal mortality } \\
\hline Deaths/1000 live births & 183 & 54 & 70.4 & 11.1 & 1 & 1 \\
\hline Total deaths & 61343 & 22813 & 62.8 & 9.0 & 2 & 4 \\
\hline Maternal mortality ratio/100 000 live births & 840 & $340 \S$ & 59.5 & 9.0 & 1 & 6 \\
\hline
\end{tabular}

*Only countries with populations greater than 500000 included.

†2009 data.

$\ddagger 2005$ data.

$\S 2010$ data. 